\title{
Short and Medium Term Variability of Emission Lines in Selected Southern Be Stars
}

\author{
Thomas Rivinius \\ Landessternwarte Königstuhl, D-69117 Heidelberg, Germany
}

\begin{abstract}
We observed a sample of several southern Be stars from 1995 to 1997 typically for several months in each season using our spectrograph HEROs. One of these stars, $\mu$ Cen, was found to be in the process of continued gradual recovery of the Hydrogen emitting disk which had been lost from 1977-1989. During the monitoring period numerous line emission outbursts were observed. A generalized pattern of an outburst cycle is derived from observations of different circumstellar lines at times of various levels of emission from the disk. Relative quiescence in which mostly periodic varaibiltiy is seen, rapid decreases of emission (precursor), outburst, and subsequent relaxation can be distinguished as the main constituing phases, even though there are distinct differences between different groups of spectral lines. Based on this empirical phenomenology, a schematic picture of the associated ejection of matter into a near-stellar orbit is sketched and similarities between $\mu$ Cen and other stars will be outlined.
\end{abstract}

\section{Observed variability}

As a part for a search for multiperiodic line profile variability (Rivinius et al., 1998a, 1998b, Štefl, this Volume, e.g.), we observed several southern stars Be stars with our fiber linked spectrograph HEROs. HEROs covers more than the entire optical range $(3450 \AA$ to $8600 \AA)$ with a resolving power of 20000 . A detailed description of the instrument is given by Kaufer (1998). The spectra were taken typically at least daily. The observations are summarized in the upper part of Table. 1.

Photospheric periods: For all four stars we found coherent photospheric periods. The multiperiodicity of $\mu$ Cen is disussed by Rivinius et al. 1998b. For $\eta$ Cen Janot-Pacheco et al. presented a poster during this meeting in which they announced several periods. At least the previously known period 0.64 day and a period of 0.57 day is also present in our data. Since the data of Janot-Pacheco et al. was taken with a entirely different sampling and equipment, we regard this as another case of a multiperiodic Be star. In the case of $\omega$ CMa only the well known 1.36 day periodicity could be confirmed. For FW CMa we were able to detect a previously unknown period of 0.83 day, behaving spectroscopically similar to $\omega \mathrm{CMa}$.

With the exception of FW CMa, these periods can also be detected in the emission. In the case of $\eta$ Cen this is clearly seen in $\mathrm{H} \alpha$, while for $\mu$ Cen this is revealed by the power distribution across the line profile, that reaches 

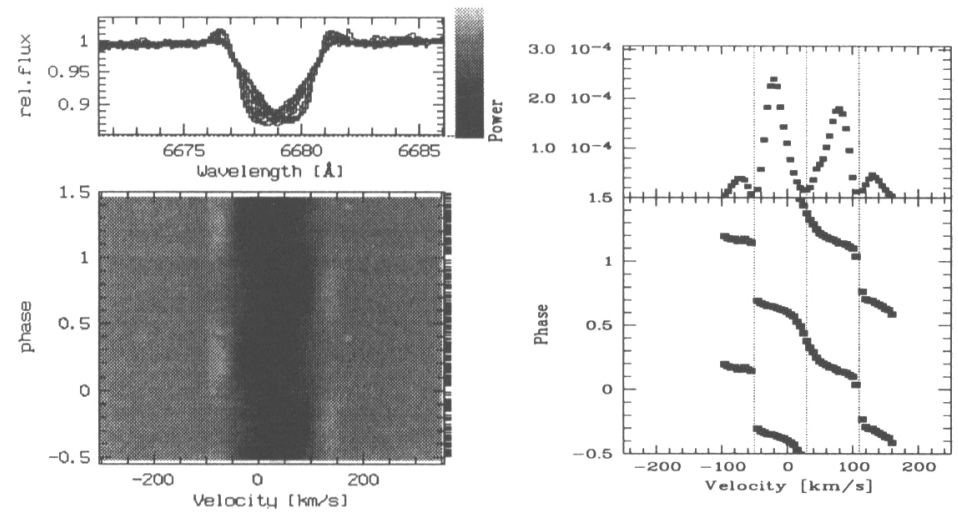

Fig. 1. The phased spectra of HeI $\lambda 6678$ of $\omega \mathrm{CMa}$ in 1997 and the results of a Fourier analysis

a secondary maximum at the positions of the emission peaks. Most clearly this behaviour however is seen in $\omega$ CMa (cf. Fig. 1).

Transient periods: For a description of this phenomenon please refer to Stefl's paper in this Volume.

Rapid decreases of emission: Just at the very beginning of an outburst, the emission peak heights of the Balmer lines in $\mu$ Cen decrease. This decrease can be as strong as $30 \%$ in $\mathrm{H} \alpha$ but less in the higher Balmer lines. Simultaneous to the peak decay however, the broad photospheric wings are filled up, so that the overall variation in equivalent width (EW) is not necessarily large. In fact, these wings increase in comparable strength in all Balmer lines, so that from $\mathrm{H} \beta$ or $\mathrm{H} \gamma$ on the EW actually decreases (counting emission as negative EW). A similar event was seen in $\omega \mathrm{CMa}$. Although the overall emission level is low in $\eta$ Cen, a decrease might be seen in our data prior to an outburst. As fourth case found by inspection of our database we mention $\kappa$ CMa.

Slow line width variations: During an outburst, the peak separation of optical thin lines like FeII $\lambda 5169$ may increase within a few days by more than $100 \mathrm{~km} / \mathrm{s}$ in the case of $\mu$ Cen. Afterwards, the peaks return to their initial positions gradually over the course of months. This behaviour has been observed also for $\eta$ Cen. There is also some indication for such an event in $\omega \mathrm{CMa}$, but due to the rather narrow lines the variations are harder to detect than for the more equator-on cases.

As summarized in Table 1, only FW CMa seems to exhibit a non-variable circumstellar environment. If this is however a true physical stability or if the variations just can't be observed under such a polar orientation because of averaging effects, or because the strong emission (the highest in our sample), or even just because it is the least intense one observed by us cannot be judged. 
Table 1. The obtained number of spectra and observed variability types

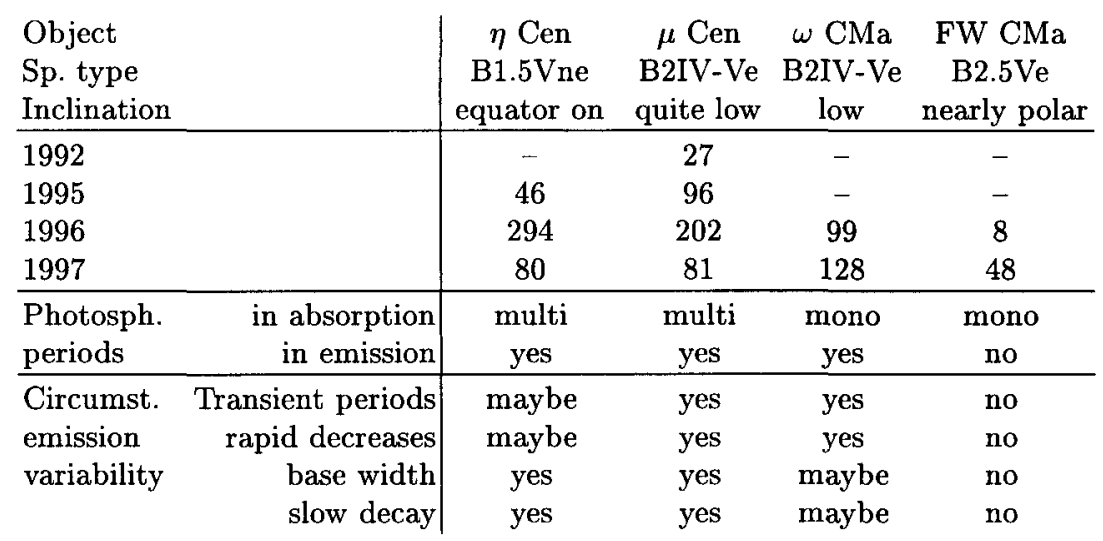

\section{The Proposed Disk Scenario}

Considering the observations above, at least for the cases of $\mu$ Cen and $\eta$ Cen we propose a, yet speculative, disk formation "fueling" scenario.

Since the base width of an optical thin emission line is given by the material closest to the star, the variability of this width indicates that the disk might be usually detached from the stellar surface. Only during outburst events, in which the disk is replenished, a considerable amount of matter is ejected to the close circumstellar environment. (Outbursts in $\mu$ Cen seem triggered by the photospheric multiperiodicity cf. e.g. Baade, this Volume). During the outbursts, one ore more clouds are ejected to close orbits, causing the transient periods. The emission height decrease/wing increase is produced by shielding of ionizing radiation towards the disk by some less dense pseudophotosphere (e.g. blue supergiant photospheres also exhibit broad emission wings in all Balmer lines, cf. e.g. Kudritzki, this Volume). After a few days, axisymmetry is attained and the ejected material (partly?) moves outwards and merges with the detached disk. During this process, the profiles return to their initial state. A more detailed description of this scenario based on $\mu$ Cen data only is given by Rivinius et al. 1998a.

\section{References}

Kaufer A., 1998, In: Reviews in Modern Astronomy 11, Schielicke R. (ed.), p. 177 Rivinius Th., Baade D., Štefl S., et al., 1998a, A\&A 333, 125

Rivinius Th., Baade D., Štefl S., et al., 1998b, A\&A 336, 177 


\section{Discussion}

G. Koenigsberger: What is the time scale over which the periods you mention are coherent? Why is an explanation in terms of the presence of a binary companion excluded? How can the mechanism you describe for the variability lead to such coherent periodicities?

T. Rivinius: At least ten years. The observed multi-periodicity is hardly explainable by a singly periodic physical process of orbital motion. I am rather optimistic for the cases of $\mu$ Cen and $\eta$ Cen that it is non-radial pulsation. For $\omega \mathrm{CMa}$ and FW CMa there is less evidence, but NRP is still a mechanism to be considered.

S. Shore: It seems that you are asking for a lot of work to be done by a little cloud: i.e., to shield a local region and then maintain shielding after spreading out. Have you calculated the optical depths of the clouds? How much mass is involved?

T. Rivinius: We have not yet modelled this hypothesis, but the broad wings observed during the initial phases of an outburst of $\mu$ Cen (cf. Rivinius et al. 1998a) might indicate a dense layer that starts to get away from the central star while in this layer temperature and density conditions as in late B supergiants prevail.

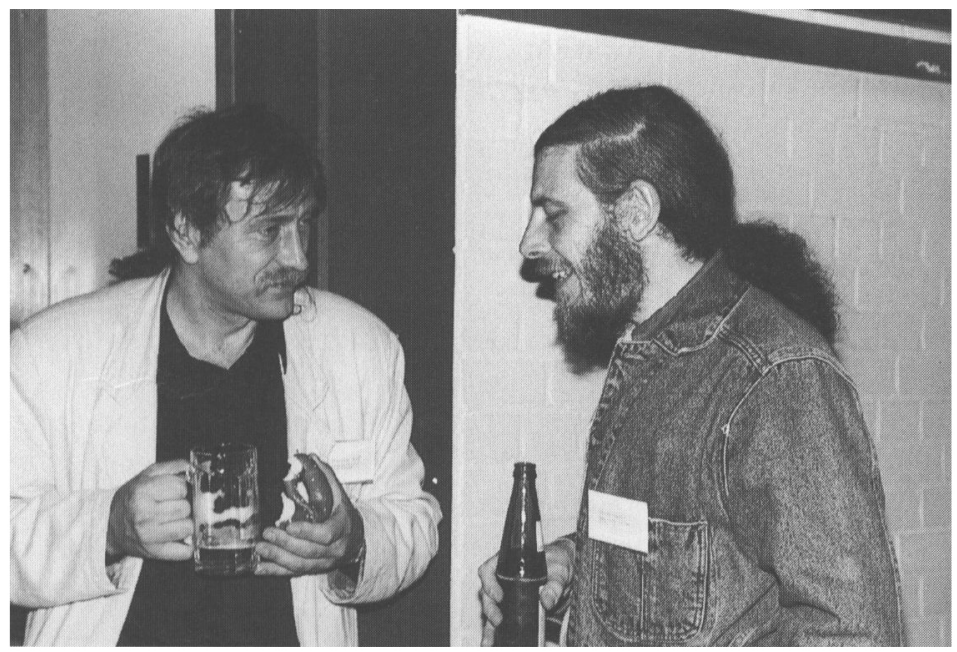

Stanislav Štefl and Steve Shore 